\title{
Core RNAi Machinery and Three Sid-1 Related Genes in Spodoptera litura (Fabricius)
}

\author{
Liang Gong ${ }^{1,2, \$}$, Zheng Wang ${ }^{1, \$}$, Huidong Wang ${ }^{1}$, Jiangwei $\mathbf{Q i}^{1}$, Meiying $\mathrm{Hu}^{{ }^{* *}}$ and Qiongbo $\mathrm{Hu}^{{ }^{* *}}$ \\ ${ }^{1}$ Key Laboratory of Pesticide and Chemical Biology, College of Natural Resources and Environment, South China \\ Agricultural University, Guangzhou 510642, Guangdong Province, China \\ ${ }^{2}$ Key Laboratory of Plant Resource Conservation and Sustainable Utilization, South China Botanical Garden, Chinese \\ Academy of Sciences, Guangzhou 510650, Guangdong Province, China \\ *For correspondence: humy@scau.edu.cn; hqbscau@126.com \\ $\S$ All authors equally contribute the research
}

\begin{abstract}
Our previous works confirmed that RNAi efficiency can be achieved by injection of dsRNA into the fourth-instar larva (Zhao et al., 2013) or pupa (Dong et al., 2013) in Spodoptera litura. However, it has been considered that the RNAi efficiency is refractory in lepidopteran insects. In the present study, we focused on the RNAi machinery in $S$. litura by using an our previously constructed transcriptome (GenBank TSA: GBBY00000000) to detect genes predicted to be involved in the RNAi response, including the R2d2, Loqs, Ago1, Ago2, Dic1 and Dic2. By using quantitative PCR, we found that these genes were highly expressed in the development stage of adult and in the tissue of fat body. We also found three homologues of the sid1-like gene responsible for dsRNA transport inside the cell in the $S$. litura transcriptome. These genes were preferentially expressed in the pupal stage and in the tissue of midgut. Z-test of neutral evolution analysis showed that only between Sl-sidl and Sl-sid3 have the probability of rejecting the null hypothesis of strict-neutrality $(\mathrm{dN}=\mathrm{dS}, \mathrm{P}<0.05)$, indicating no evolutionary pressure between Sl-sidl and Sl-sid3. Our data support that $S$. litura may have a conserved RNAi mechanism, but that different tissues and/or developmental stages may differ in the efficacy of the RNAi response. (C) 2015 Friends Science Publishers
\end{abstract}

Keywords: Spodoptera litura; RNAi; RNAi machinery; Sid; Systemic RNAi response

\section{Introduction}

RNA interference (RNAi) was firstly described in petunias at the early $1990 \mathrm{~s}$, in which genes were over expressed for pigment production, but presenting white flowers that resulted from pigment synthesis inhibition, caused by coordinated gene silencing of both transgene and endogenous gene (Napoli et al., 1990). This phenomenon was known as "co-suppression" and also observed in other species of plants, fungi and insects, but the mechanism leading to gene silencing is still not fully understood, especial in lepidopteran insects.

Currently there is no ideal experimental strategy for successful application of RNAi in the study of insect functional genes, probably due to the limited understating of the molecular basis of the RNAi mechanism in insects (Scott et al., 2013). The core RNAi machinery in some organisms, such as Drosophila melanogaster consists of two RNAi pathways: the small interfering RNA (siRNA) and the micro RNA (miRNA) pathways. Two major functions of the RNAi pathways in some organisms have been confirmed: (1) to regulate gene expression and control critical cellular and developmental processes; (2) to protect from viral infection (Ding and Voinnet, 2007; Castel and Martienssen, 2013). A number of key protein components involved in the RNAi pathways have been reported in plant and invertebrates mainly including Dicer (Dic), R2D2, Loquacious (Loq) and Argonaute (Argo) (Liu et al., 2003; Liu et al., 2006; Makarova et al., 2009; Swevers et al., 2011; Wu et al., 2013). As reported in D. melanogaster that the proteins Dic1, Loq and Ago1 are involved in the miRNA pathway whereas Dic2, R2d2 and Ago2 are involved in the RNAi pathway (Mukherjee and Hanley, 2010). Through these proteins, the stem-loop hairpin transcripts of endogenous genes are processed to generate miRNAs or long dsRNA precursors (either exogenous or endogenous) that are cut to siRNAs. Both of the miRNA and siRNA are incorporated into the RNA-induced silencing complex (RISC) to generate miRISC and siRISC, respectively. The effectors (miRISC and siRISC) serve as guide RNA for sequence-specific cleavage and/or translational repression of complementary mRNA (Bernstein et al., 2001; Hannon, 2002; Richter et al., 2013). However, the core RNAi machineries not include genes for a systemic RNAi response, when the RNAi effect spreads to the whole body from the initial silenced cell, as it was found in 
Caenorhabditis elegans. Several genes have been identified in C. elegans that are crucial for systemic spread of dsRNA, but not for the interference itself, such as sid-1, encoding a multi-trans membrane domain protein that fucntions as a channel for dsRNA (Winston et al., 2002; Winston et al., 2007). Interestingly, a systemic RNAi response does not exist in $D$. melanogaster, which may be caused by the absence of sid-1 in this species (Tomoyasu et al., 2008). Insect Sid-1 genes were studied in the preliminary stage, although its homologs have been found in various insect orders (Tian et al., 2009; Luo et al., 2012; Bansal and Michel, 2013).

The tobacco cutworm, Spodoptera litura Fabricius (Lepidoptera: Noctuidae) is one of the most destructive insect pests of agricultural crops, such as cotton, vegetables, oilseed and fiber crops. In recent years, its frequent outbreaks in the Asian have caused considerable agricultural economic losses and long-term use of synthetic pesticides that have resulted in serious resistance of $S$. litura against various insecticides (Tuan et al., 2013). Therefore, S. litura has been paid close attention with the studies extensive on its insecticidal toxicology (Gong et al., 2014; Meng et al., 2013), molecular mechanisms of its insecticide resistance (Rajagopal et al., 2002; Zhou et al., 2012) and new alternatives for its integrated management (Arasu et al., 2013; Huang et al., 2013; Zhao et al., 2013). In some of these cases, it was proofed that RNAi is a useful tool for studying genes function in $S$. litura. In addition, our previous works confirmed as well that RNAi efficiency can be achieved by injection of dsRNA into the fourth-instar larva (Zhao et al., 2013) and the pupa (Dong et al., 2013) in S. litura. However, it was known that the RNAi efficiency is refractory in lepidopteran insects (Swevers et al., 2013). In the present study, with the aim to search the limiting factors that may affect the efficiency of RNAi in S. litura, we explored the RNAi machinery and sid-1 homologs from our previously constructed $S$. litura transcriptome. We found that the homologues of RNAi machinery are highly conserved in $S$. litura compared to alternative invertebrate systems.

\section{Materials and Methods}

\section{Insect Strain and Samples Collection}

A population of Spodoptera litura that has been reared in our lab for more than 10 years was kept under the conditions as previously described (Tu and Zheng, 2010). Four insect samples from different developmental stages including eggs, larvae (the third instar), pupae and adults (male and female) were collected during $24 \mathrm{~h}$ after metamorphosis or molt. Tissue samples including midgut, cuticle and fat body were dissected from the fifth stage larvae of $S$. litura. All the samples were kept in $-80^{\circ} \mathrm{C}$ until to be used. Annotation and Bioinformatics Analysis of Dcr, R2d2,
Loq, Ago and Sids in S. litura Transcriptome

cDNAs coding for Dcr, R2d2, Loq, Ago and Sids were retrieved from the functional annotation of $S$. litura transcriptome (GenBank TSA: GBBY00000000). Each contig displaying significant similarity to $D c r(D c r l$ and Dcr2), R2d2, Loq, Ago (Agol and Ago2) and Sid (Sidl, Sid2 and Sid3) homologs was further performed ORF (Open Reading Frame) search at NCBI on-line service (http://www.ncbi.nlm.nih.gov/gorf/gorf.html). More sequence analysis was performed by several on-line tools, such as multiple sequence alignment was carried out with the online service at http://www.ebi.ac.uk/Tools/msa/clustalw2/; Domain architecture was predicated by Scan-Prosite (http://prosite.expasy.org/scanprosite/) (De et al., 2006); prediction of trans membrane helices was performed by online serves of TMpred program (http://www.ch.embnet.org/software/TMPRED_form.html) (Ikeda et al., 2003). The phylogenetic tree was constructed using MEGA 4.0 software with Neighbor-joining method (Tamura et al., 2007). Z-test of neutral evolution analysis among the nucleotide sequence of three $S$. litura sids was computed using the Nei-Gojobori method (Nei and Gojobori, 1986).

\section{Quantitative RT-PCR Analysis of Core RNAi Machineries and Three SID-related Genes in S. litura Different Tissues and Developmental Stages}

Quantitative RT-PCR (qRT-PCR) was performed to verify the expression profile of the selected genes in tissues of the firth stage larva of $S$. litura including midgut, cuticle and fat body, as well as different developmental stages including egg, the third instar larva, pupa and adult. Experiments including RNA extraction, DNase treatment, cDNA synthesis and qPCR reactions were performed as described previously (Gong et al., 2011; Gong et al., 2013).

qRT-PCR reactions were performed by three technical replicates on a BioRad QQ5 real-time PCR detection system using $200 \mathrm{ng}$ of cDNA, $0.2 \mu \mathrm{M}$ of primers and SYBR Premix Ex Taq (TaKaRa). The primers were listed in Table 1. Amplification conditions consisted of an initial denaturation at $95^{\circ} \mathrm{C}$ for 30 s followed by 40 cycles of $95^{\circ} \mathrm{C}$ for $5 \mathrm{~s}, 58^{\circ} \mathrm{C}$ for $30 \mathrm{~s}$ and a dissociation step was added as the end. After the reaction was completed, analysis of the amplification and melting curves was performed according to the manufacturer's instructions. The relative amounts of the transcripts were first normalized to the endogenous reference gene and then normalized relative to the transcripts level in stage of egg or in the tissue of midgut of $S$. litura according to the $2^{-\Delta \Delta \mathrm{Ct}}$ method (Livak and Schmittgen, 2001).

\section{Statistical Analysis}

qRT-PCR data are represented by the mean SEM. 
Statistical Analysis was performed based on t-test with $\mathrm{p}<0.05$ representing significance using Sigma Plot 12.0 software (Systat Software Inc.).

\section{Results}

Identification of Three SID-related Genes and Phylogenetic Relation Analysis in S. litura

Three unigenes coding SID-related genes were retrieved from the S. litura transcriptome (Table 2). The Comp16495 contig (GenBank KF717090) was 2,097 bp long, with an open reading frame encoding 680 amino acids. Sequence analysis indicates that this transcript shares $88 \%$ and $84 \%$ similarity to Helicoverpa armigera sid-1 (KC292008) and Bombyx mori sid-3 (NM_001113265), respectively. The Comp16844 contig GenBank KF717089) was 2,373 bp long, with an open reading frame encoding 789 amino acids. Sequence analysis indicates that this transcript shares $75 \%$ similarity to Bombyx mori sid-1 (NM_001113264). On the other hand, contig Comp18252 (GenBank KF717088) was 2,649 bp long and has an open reading frame encoding 873 amino acids. Sequence analysis indicated that this transcript shares $73 \%$ similarity to Bombyx mori sid-2 (XM_004930678). The prediction graphics of the three S1SIDs showed the number of transmembrane regions are 13, 11 and 10, respectively in SL-SID1, SL-SID2 and SL-SID3 (Supplementary file 1). Codon-based test of neutrality analysis among three sid-1 orthologs of $S$. litura showed that the only probability of rejecting the null hypothesis of strict-neutrality occurs between Sl-Sid1 and Sl-Sid3 $\left(\mathrm{d}_{\mathrm{N}}=\right.$ $\mathrm{d}_{\mathrm{s}} ; \mathrm{P}<0.05$ ), suggesting no evolutionary pressure between the sequences of Sl-Sidl and Sl-Sid3. A phylogenetic tree was generated using 18 code regions of SID-related proteins from 11 species including vertebrates, insects and $C$. elegans (Fig. 2). The phylogenetic tree clearly shows that SID-related proteins have long-time independent evolutionary history.

\section{Characterizations of Two dicer genes in S. litura}

Contig S1-Dicer-1 (GenBank KF717091, Table 2) was 4,140 bp and encoded a 1,362 amino acid protein. Sequence alignment shows that this contig has $53.6 \%$ and $29.52 \%$ similarity to Bombyx mori Dicer-1 (XP_004922366) and Drosophila melanogaster Dicer-1 (ABD61603), respectively. We found a highly conserved region among these three species, which ranges from 825-944 in Sl-Dicer1 , but no function has been reported about this region yet (Supplementary file 2A). A second contig, Sl-Dicer-2 (GenBank KF717092, Table 2), was 5,112 bp in length and encoded a protein of 1,685 amino acids. Sequence alignment shows that SL-Dicer-1 has $63.74 \%$ and 27.83 similarity to Bombyx mori Dicer-2 (NP_001180543) and Drosophila melanogaster Dicer-2 (ABB54749). We found a dsRNA-specific ribonuclease region (amino acids 1207-1273)

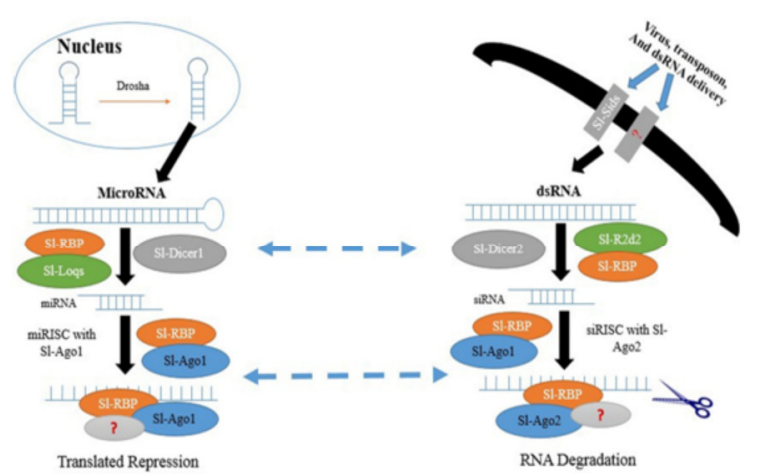

Fig. 1: Diagram of the pathways leading to RNA interference by microRNAs and siRNAs in $S$. litura. (siRISC: RNA Induced Silencing Complex associated with siRNA; miRISC: miRNA associated RISC; Ago: Argonaute; Dcr: Dicer; Loquacious: Loqs; RBP: RNAbinding protein)

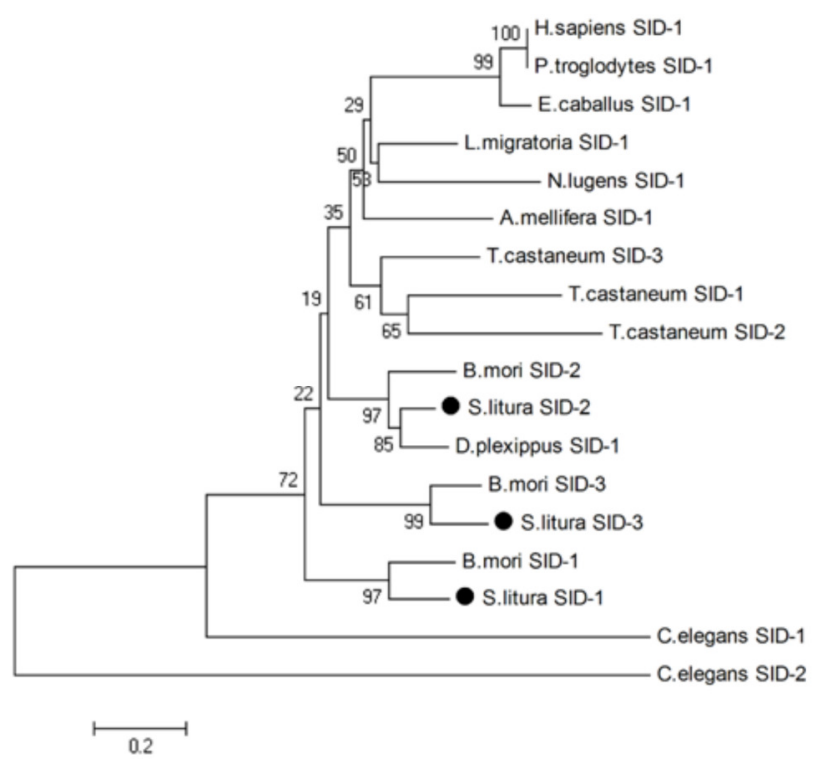

Fig. 2: Unrooted phylogenetic tree of SIDs from vertebrates, insects and C.elegans generated by the Neighbor-joining method using the software package MEGA4 (Tamura et al., 2007)

The SIDs included in the analysis were Homo sapiens (NP_060169); Pan troglodytes (XP_526266); Equus caballus (XP_001917509); Locusta migratoria (AFQ00936); Nilaparvata lugens (AEI25514); Apis mellifera (XP_395167); Tribolium castaneum SID-1 (NP_001099012); Tribolium castaneum Sid-2 (NP_001103253); Tribolium castaneum SID3(NP_001099128); Bombyx mori SID-1 (NP_001106735); Bombyx mori SID-2 (BAF95807); Bombyx mori SID-3 (NP_001106736); Danaus plexippus (EHJ72579); Caenorhabditis elegans SID-1(NP_504372); Caenorhabditis elegans SID-2 (NP_499823)

in B. mori Dicer-1 (Marchler-Bauer et al., 2013), which was highly conserved in S. frugiperda Sl-Dicer-2 $\left({ }^{1218}\right.$ LGDSF $^{1223}$ and ${ }^{1254} \mathrm{SNRNL}^{1257}$ ), indicating that they may represent active sites of dsRNA-specific ribonuclease in SL-Dicer-2 (Supplementary file 2B). A phylogenetic tree based on 
Table 1: Primers used in qRT-PCR

\begin{tabular}{|c|c|}
\hline Name/Primer Sequence & size(bp) \\
\hline \multicolumn{2}{|l|}{ SL-Dcr } \\
\hline SL-Dcr1_Fw: 5' TGCTGTATGCGATAGTATCA 3' & 473 \\
\hline SL-Dcr1_Rv: 5'CTCTTCCGTATGTTGGTATATG 3' & \\
\hline SL-Dcr2_Fw: 5' GGACGAGTATGTAGAGGATG 3' & 212 \\
\hline SL-Dcr2_Rv: 5' CAGTGTTGACCAGGAAGAA 3' & \\
\hline SL-Ago SL-Ago1_Fw: 5'ACTTCGCCACAGACCTTA 3' & 478 \\
\hline SL-Ago1_Rv: 5'CCGCCTCCAACTTGATAC 3' & \\
\hline SL-Ago2_Fw: 5' AGTCGTGCTTCGTCTCTA 3' & 461 \\
\hline SL-Ago2_Rv: 5' CCGTCTGATTCCGTAATAGT 3' & \\
\hline SL-R2D2 R2D2_Fw: 5'GAGCTGTGCGAGGAATAC 3' & 336 \\
\hline $\begin{array}{l}\text { R2D2_Rv: 5'AATGAGTGCGAGGTGGTA 3' SL-Loq SL-Loq_Fw: 5' ACAAGTCACAGCCACAAG 3'SL-Loq_Rv: 5' } \\
\text { AAGTCCTGAGCCATAGCA 3' }\end{array}$ & 255 \\
\hline \multicolumn{2}{|l|}{ SL-sid } \\
\hline SL-sid1_Rv: 5'TCCTCTCAGACTTCAACCA3' & 267 \\
\hline SL-sid1_Fw: 5' CCATCACCAACACTGCTAT3' & \\
\hline SL-sid2_Rv: 5'GCTACTTCAACTTCCTATGC3' & 133 \\
\hline SL-sid2_Fw: 5'TGATGCGTATTATGTCTCCT3' & \\
\hline SL-sid3_Fw: 5' AACGCACTCAACTTCCAA 3' & 192 \\
\hline SL-sid3_Rv: 5'GAACACGCTCCAGAACAA3'Reference geneS. litura actin_Fw:5' GCCAACAGGGAGAAGATG 3'S. & 230 \\
\hline
\end{tabular}

Table 2: Description of RNAi machinery and three sid-1 related genes in S. litura

\begin{tabular}{|c|c|c|c|c|c|c|c|c|}
\hline$\overline{\text { Name }}$ & $\overline{c D N A}(b p)$ & ORF & Accession numbers & amino acid(aa) & $\overline{\mathrm{pI}}$ & $\mathrm{MM}(\mathrm{kDa})$ & Match & $\overline{\text { Identities }}$ \\
\hline S1-R2d2 & 1029 & $22-109$ & KF717086 & 335 & 6.004 & 36.695 & Bombyx mori(NP_001182007) & $50 \%$ \\
\hline S1-Loqs & 1188 & $31-1188$ & KF717087 & 385 & 8.766 & 42.511 & Danaus plexippus(EHJ63609) & $92 \%$ \\
\hline Sl-Ago1 & 2805 & $19-2805$ & KF717084 & 928 & 9.024 & 103.22 & Danaus plexippus (EHJ71131) & $94 \%$ \\
\hline S1-Dic1 & 4140 & $55-4140$ & KF717091 & 1362 & 5.008 & 153.977 & Bombyx mori(XP_004922366) & $61 \%$ \\
\hline S1-Dic2 & 5112 & $55-5112$ & KF717092 & 1685 & 6.288 & 192.769 & Bombyx mori(NP_001180543) & $64 \%$ \\
\hline Sl-Sid1 & 2373 & 4-2373 & KF717089 & 789 & 7.843 & 88.471 & Bombyx mori(NP_001106735) & $63 \%$ \\
\hline S1-Sid2 & 2649 & $28-2649$ & KF717088 & 873 & 6.498 & 99.456 & Danaus plexippus (EHJ69120) & $66 \%$ \\
\hline S1-Sid3 & 2097 & $25-2067$ & KF717090 & 680 & 7.93 & 75.388 & Bombyx mori(NP_001106736) & $63 \%$ \\
\hline
\end{tabular}

Note: ORF, Open reading frame; MM, Molecular mass; $\mathrm{p} I$, Isoelectric Point

amino acid sequences of Dicer genes was constructed using neighbor-joining method with 1,000 replications (Supplementary file 2C), which showed two groups reflecting the functional divergence of Dicer-1 for producing MicroRNA and Dicer-2 for producing siRNA as in D. melanogaster (Lee et al., 2004).

\section{Identification of Two Argonaute (Argo) Genes in $S$. litura}

The contig Sl-Ago1 (GenBank KF717084, Table 2) was $2,805 \mathrm{bp}$ long, with an open reading frame encoding 928 amino acids. Multiple sequence alignment shows that SLAgo 1 has $96.6 \%, 95.9 \%$ and $85.1 \%$ similarity to $B$. mori (NP_001095931), D. plexippus (EHJ71131) and D. melanogaster (NP_523734) Argol, respectively. In contrast, contig SL-Argo2 (GenBank KF717085, Table 2) was 3,273 bp and encoded 1,061 amino acids. Multiple sequence alignment shows that Sl-Argo2 has 53.6\%, 64.9\% and $31.4 \%$ similarity to B. mori Argo 2 (NP_001036995), D. plexippus (EHJ72821) and D. melanogaster (ABB54726), respectively.

\section{Sequence Analysis of $R 2 d 2$ and loquacious in S. litura}

A 1,029 bp full-length cDNA of R2D2 (GenBank
KF717086, Table 2) was found in the $S$. litura transcriptome, which has a code region of $1,008 \mathrm{bp}$ encoding 335 amino acids. Multiple sequence alignment shows that Sl-R2d2 has $46.9 \%$ and $20 \%$ similarity to $B$. mori R2d2 (NP_001182007) and Tribolium castaneum R2d2 (NP_001128425), respectively. The architecture of Sl-R2d2 indicates two regions with the function of double stranded RNA-binding, which are from the amino acids 3 (T) to 70 (R) and from 103 (S) to 171 (E) (Fig. 4, Supplementary file 3). A 1,188 bp full-length cDNA of Loqs (GenBank KF717087, Table 2) was also found in the $S$. litura transcriptome, which has a code region of $1,158 \mathrm{bp}$ encoding 385 amino acids. Multiple sequence alignment shows that Sl-Loqs has $83.5 \%$ and $58.1 \%$ similarity to $B$. mori Loqs (NP_001182008) and Tribolium castaneum Loqs (XP_966668), respectively. The architecture of S1-Loqs indicates three regions with the function of double stranded RNA-binding, which are from the amino acids 75 (T) - 146 (G), 175 (N) - 247 (D) and 313 (N) - 381 (I) (Fig. 4, Supplementary file 3$)$.

Expression Profiles of $S$. litura SID-related Genes in Different Tissues and Developmental Stages of $S$. litura

We found that three $S$. litura Sid-related genes were all 
expressed in tissues of the fifth instar larvae, including midgut, cuticle and fat body. However, the highest levels of expression were detected in the midgut and the lowest were detected in the cuticle (Fig. 5B). For example, expression of S. litura sid-3 in midgut was $>1,000$-fold and 47.6-fold than that detected in the cuticle and fat body, respectively. This observation indicates a possible role of Sl-Sid3 with the function of uptake of dsRNA in $S$. litura midgut. Expression of these genes were tested in different developmental stages, showing that the highest expression occurs in pupa. Moreover, the expression of Sl-sidl and Sl-sid2 were significantly higher in the egg stage than in other stages (Fig. 5D).

\section{Expression Profiles of Genes Associated with the RNAi Machinery in Different Tissues and Developmental Stages of $S$. litura}

We found that all the selected contigs (Sl-R2d2, Sl-Ago, SlLoqs and Sl-Dic) were expressed in tissues of midgut, cuticle and fat body. The highest expression levels for all of them were detected in the fat body, followed by in the cuticle, except that $S L-D c r 2$ was higher expressed 3.2-fold in the midgut than the in cuticle (Fig. 5A). The expression in developmental stages including larva, pupa and adult, showed that their expression increased from larva to pupa, and pupa to adult. For example, the expression of Sl-Loq was 1.6-fold and 5.6-fold higher in the pupa and adult stages, respectively, than the expression in the larva stage (Fig. 5C).

\section{Discussion}

In the present study, the core RNAi machineries and sid-1 related genes were found in $S$. litura transcriptome, indicating that $S$. litura possesses all of the core RNAi machinery genes including R2d2, Loqs, Ago1, Ago2, Dicl and Dic2, as well as three sid homologs for the possible function of spreading double-strand RNA among cells or/and tissues. Our data support that $S$. litura may have systemic RNAi response, but different tissues and/or developmental stages may differ in the efficiency of the RNAi response. Our work also the first point out that two RNA pathways (siRNA and miRNA pathways, Fig. 1) exist in $S$. litura, suggesting that $S$. litura may have a conserved RNAi mechanism.

General speaking, Sl-Dic1, Sl-Loqs and Sl-Ago1 are involved in the miRNA pathway whereas Sl-Dic2, Sl-R2d2 and Sl-Ago2 are involved in the siRNA pathway. But it should be noted that there is extensive interaction and overlap between the two branches (Represented by dotted arrows in Fig. 1). The protein architectures of both Sl-Argo1 and Sl-Argo2 consisted of PAZ and PIWI domains (Fig. $3 \mathrm{~A}$ ), which are typical of proteins involved in posttranscriptional gene silencing (PTGS) through RNAi (Cerutti et al., 2000). Although, Sl-Argo1 and Sl-Argo2 are

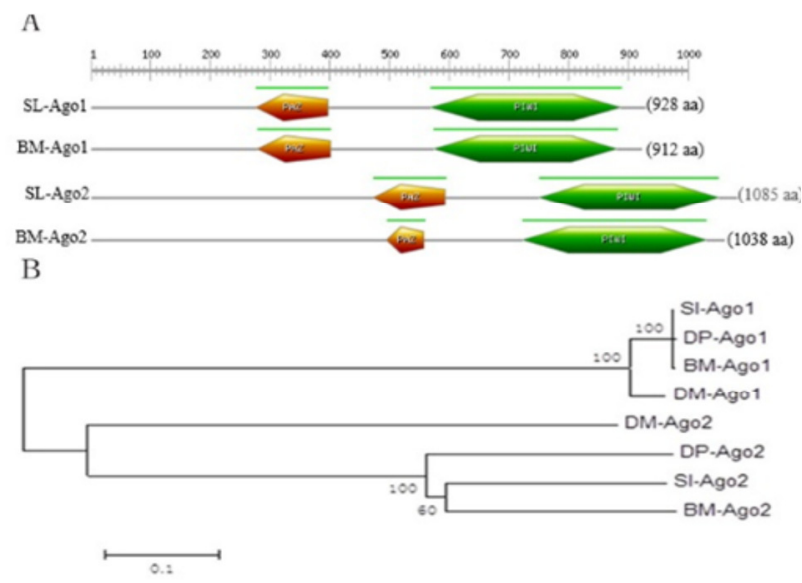

Fig. 3: Protein architectures (A) and phylogenetic analysis (B) of Sl-Ago1 and Sl-Ago2. Abbreviations are DP, Danaus plexippus; BM, Bombyx mori

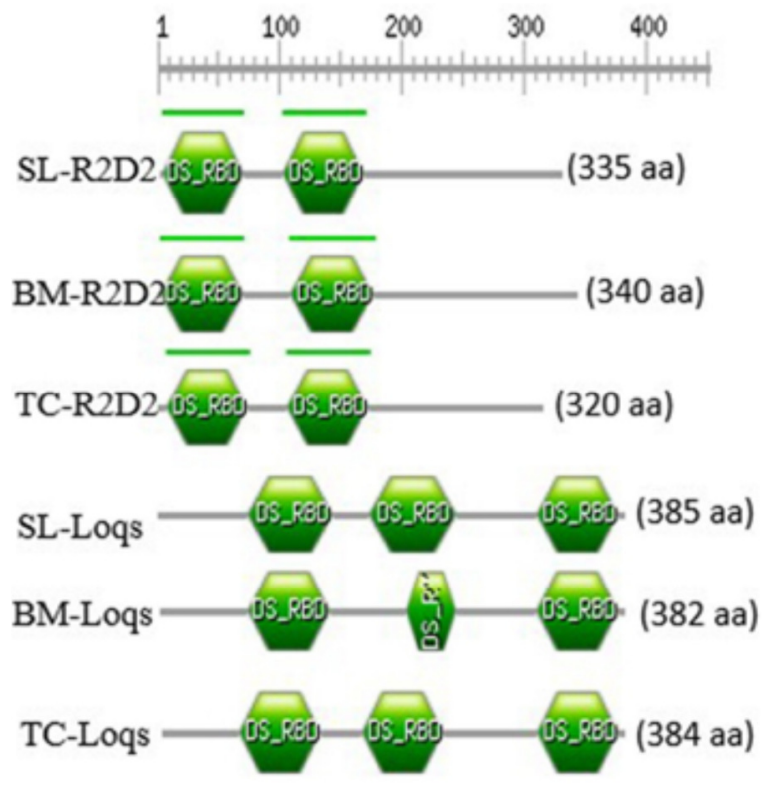

Fig. 4: Protein architectures of S1-R2d2 and S1-Loqs. Abbreviations are TC, Tribolium castaneum; BM, Bombyx mori

highly conserved in PAZ and PIWI domains, the phylogenetic analysis indicates they belong to two different groups within Lepidoptera ( $S$. litura, B. mori and $D$. plexippus) (Fig. 3B), suggesting both are especial binding modules that accommodate the small RNA components, such as microRNAs and siRNAs (Meister and Tuschl, 2004). Both R2d2 and loqs are functional in dsRNAbinding, but they perform different biochemical functions, for example, Loqs is required for processing of miRNA precursors by Dcr-1, while $\mathrm{R} 2 \mathrm{~d} 2$ is required for Dcr-2 dependent loading of siRNAs into Ago2 (Marques et al., 2010). All of the core RNAi machineries were highly expressed in the fat body, which may be the main tissue 

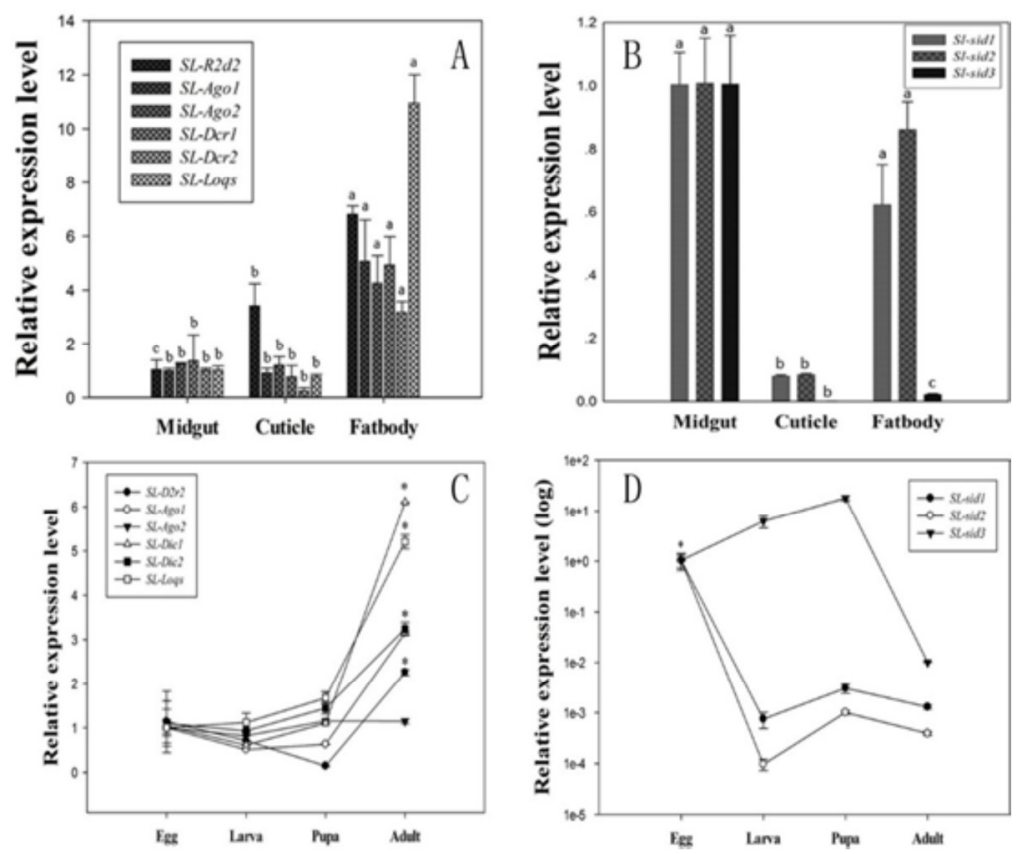

Fig. 5: Relative transcript quantification of gens involved in the RNAi response in different developmental stages and different tissues. Bars with same letter are not significantly different from each other at $\mathrm{P}<0.05$ based on T-test $(* \mathrm{p}<0.05)$

for producing proteins involved in the RNAi response. On the other hand, a limited amount of selected genes were also detected in midgut and cuticle, indicating the fifth instar $S$. litura larvae may possess the RNAi machinery in the whole-body. In the present study, the adult is the high expression stage of the RNAi machineries among different developmental stages, however, in the case of A. glycines, the core RNAi Machineries, such as Dcr2, Ago2 and R2d2 were high expression in the early development stages (Bansal and Michel, 2013). In four reproductive morphs of Acyrthosiphon pisum, expression levels of four genes (two Argonaute genes and two dicer genes) were the highest in parthenogenetic sexuparae female compared to parthenogenetic virginoparae female, sexual oviparae female and sexual male (Ortiz-Rivas et al., 2012).

We supposed that the SID-related proteins may evolve from one common ancient gene, but there was diverged during the evolutionary history. For example, sid-1 participates in the systemic RNAi in C. elegans (Grishok, 2005) but this function has been lost following the evolution in Locusta migratoria (Luo et al., 2012). Lepidopterna insects ( $S$. litura and B. mori) seem to be located in the upstream of the evolution indicating a possible role in the systemic RNAi response (Fig. 2). However, because of the small amount of data available, it is difficult to analyze the evolution and duplication of sid-1-like genes in different organisms. The sid-1 gene or thologous were reported in many different insect orders, but few studies focus on its expression and functionality (Huvenne and Smagghe, 2010). In a study of Aphis glycines sidl, which was not significant differences in the expression level in different tissues, such as epidermis, gut and fatbody (Bansal and Michel, 2013). However, our work confirmed that three sid1 gene or thologs in $S$. litura are significantly high expression in the midgut, indicating a possible role of the sid1-like genes in uptake dsRNA in the midgut lumen of $S$. litura. Although sid-1 homologs were found in many different insect species, no conclusion could be drawn regarding these gene orthologs in the involvement of dsRNA uptake. For example, three sid-1 or thologs of Tribolium castaneum were silenced individually or all together, but no influence of RNAi in this species was found (Tomoyasu et al., 2008). Our data for the first time provide the genes sequence information of three sid-1 or thologs in $S$. litura, which will facilitate the detailed function research of sid-1 or thologs in a non-model organism of S. litura.

\section{Conclusion}

We studied the RNAi machinery in S. litura by using a transcriptome to detect genes predicted to be involved in the RNAi silencing response, including the $R 2 d 2$, Loqs, Agol, Ago2, Dicl and Dic2 genes. All these genes were highly expressed in the tissue of the adult fat body. Our data support that $S$. litura may have a conserved RNAi mechanism, but that different tissues and/or developmental stages may differ in the efficacy of the RNAi response. We also found three homologues of the SID-1 gene responsible for dsRNA transport inside the cell in $S$. litura transcriptome. These genes were preferentially expressed in the midgut tissue, indicating $S$. litura might possess systemic RNAi response. By Z-test of neutral evolution 
analysis, there is no evolutionary pressures occurred between Sl-sidl and Sl-sid3. Based on their preferentially expressed tissue and developmental stage, our data are conclusive that injecting of dsRNA into pupa or adult of $S$. litura that is more possible to obtain the efficacy of the RNAi response in this species.

\section{Acknowledgement}

The work was supported by grants from National Hi-tech Research and Development (863) Program of China (2012AA101505) and National Natural Science Foundation of China (31171870).

\section{References}

Arasu, M.V., N.A. Al-Dhabi, V. Saritha, V. Duraipandiyan, C. Muthukumar and S.J. Kim, 2013. Antifeedant, larvicidal and growth inhibitory bioactivities of novel polyketide metabolite isolated from Streptomyces sp. AP-123 against Helicoverpa armigera and Spodoptera litura. BMC Microbiol., 13, doi:10.1186/1471-2180-13105

Bansal, R. and A.P. Michel, 2013. Core RNAi Machinery and Sid1, a Component for Systemic RNAi, in the Hemipteran Insect, Aphis glycines. Int. J. Mol. Sci., 14: 3786-3801

Bernstein, E., A.A. Caudy, S.M. Hammond and G.J. Hannon, 2001. Role for a bidentate ribonuclease in the initiation step of RNA interference. Nature, 409: 363-366

Castel, S.E. and R.A. Martienssen, 2013. RNA interference in the nucleus: roles for small RNAs in transcription, epigenetics and beyond. Nat. Rev. Genet., 14: 100-112

Cerutti, L., N. Mian and A. Bateman, 2000. Domains in gene silencing and cell differentiation proteins: the novel PAZ domain and redefinition of the Piwi domain. Trends Biochem. Sci., 25: 481-482

De, C.E., C.J.A. Sigrist, A. Gattiker, V. Bulliard, P.S. Langendijk-Genevaux, E. Gasteiger, A. Bairoch and N. Hulo, 2006. ScanProsite: detection of PROSITE signature matches and ProRule-associated functional and structural residues in proteins. Nucleic Acids Res., 34: W362-W365

Ding, S.W. and O. Voinnet, 2007. Antiviral immunity directed by small RNAs. Cell, 130: 413-426

Dong, X.L., G.H. Zhong, M.Y. Hu, X.Yi, H.M. Zhao and H.D. Wang, 2013. Molecular cloning and functional identification of an insect odoran receptor gene in Spodoptera litura (F.) for the botanical insecticide rhodojaponin III. J. Insect Physiol., 59: 26-32

Gong, L., H.D. Wang, J.F. Huang, M.Y. Hu, Z. Hu and G.H. Zhong, 2014. Camptothecin- induced expression of programmed cell death gene 11 in Spodoptera litura. Pest Manage. Sci., 70: 603-609

Gong, L., X.Q. Yang, B.L. Zhang, G.H. Zhong and M.Y. Hu, 2011. Silencing of Rieske iron-sulfur protein using chemically synthesised siRNA as a potential biopesticide against Plutella xylostella. Pest Manage. Sci., 67: 514-520

Gong, L., Y. Chen, Z. Hu and M.Y. Hu, 2013. Testing insecticidal activity of novel chemically synthesized siRNA against Plutella xylostella under laboratory and field conditions. PLoS One, 8: e62990

Grishok, A., 2005. RNAi mechanisms in Caenorhabditis elegans. FEBS Lett., 579: 5932-5939

Hannon, G.J., 2002. RNA interference. Nature, 418: 244-251

Huang, S.H., J.D. Xian, S.Z. Kong, J.H. Xie, J. Lin, J.N. Chen, H.F. Wang and Z.R. Su, 2013. Insecticidal activity of pogostone against Spodoptera litura and Spodoptera exigua (Lepidoptera: Noctuidae). Pest Manage. Sci., doi: 10.1002/ps.3635

Huvenne, H. and G. Smagghe, 2010. Mechanisms of dsRNA uptake in insects and potential of RNAi for pest control: A review. J. Insect Physiol., 56: 227-235
Ikeda, M., M. Arai, T. Okuno and T. Shimizu, 2003. TMPDB: a database of experimentally-characterized transmembrane topologies. Nucleic Acids Res., 31: 406-409

Lee, Y., M. Kim, J. Han, K.H. Yeom, S. Lee, S.H. Baek and V.N. Kim, 2004. MicroRNA genes are transcribed by RNA polymerase II. Embo J., 23: 4051-4060

Liu, Q.H., T.A. Rand, S. Kalidas, F.H. Du, H.E. Kim, D.P. Smith and X.D. Wang, 2003. R2D2, a bridge between the initiation and effector steps of the Drosophila RNAi pathway. Science, 301: 921-1925

Liu, X., F. Jiang, S. Kalidas, D. Smith and Q.H. Liu, 2006. Dicer-2 and R2D2 coordinately bind siRNA to promote assembly of the siRISC complexes. RNA, 12: 1514-1520

Livak, K.J. and T.D. Schmittgen, 2001. Analysis of relative gene expression data using real-time quantitative PCR and the $2^{-\Delta \Delta C t}$ method. Methods, 25: 402-408

Luo, Y., X.H. Wang, D. Yu and L. Kang, 2012. The SID-1 double-stranded RNA transporter is not required for systemic RNAi in the migratory locust. RNA Biol., 9: 663-671

Makarova, K.S., Y.I. Wolf, J. Van der Oost and E.V. Koonin, 2009. Prokaryotic homologs of Argonaute proteins are predicted to function as key components of a novel system of defense against mobile genetic elements. Biol. Direct, 4: 29

Marchler-Bauer, A., C.J. Zheng, F. Chitsaz, M.K. Debyshire, L.Y. Geer, R.C. Geer, N.R. Gonzales, M. Gwadz, D.I. Hurwitz, C.J. Lanczycki, F. Lu, S.N. Lu, G.H. Marchler, J.S. Song, N. Thanki, R.A Yamashita, D.C. Zhang and S.H. Bryant, 2013. CDD: conserved domains and protein three-dimensional structure. Nucleic Acids Res., 41: $348-352$

Marques, J.T., K. Kim, P.H. Wu, T.M. Alleyne, N. Jafari and R.W. Carthew, 2010. Loqs and R2D2 act sequentially in the siRNA pathway in Drosophila. Nat. Struct. Mol. Biol., 17: 24-30

Meister, G. and T. Tuschl, 2004. Mechanisms of gene silencing by doublestranded RNA. Nature, 431: 343-349

Meng, X., J.J. Hu, X.X. Xu, Z.Q. Wang, Q.B. Hu, F.L. Jin and S.X. Ren, 2013. Toxic tffect of destruxin A on abnormal wing disc-like (SLAWD) in Spodoptera litura Fabricius (Lepidoptera: Noctuidae). PLoS ONE, 8: e57213

Mukherjee, S. and K. Hanley, 2010. RNA interference modulates replication of dengue virus in Drosophila melanogaster cells. BMC Microbiol., 10: 127

Napoli, C., C. Lemieux and R. Jorgensen, 1990. Introduction of a chimeric chalcone synthase gene into petunia results in reversible cosuppression of homologous genes in trans. Plant Cell, 2: 279-289

Nei, M. and T. Gojobori, 1986. Simple methods for estimating the numbers of synonymous and nonsynonymous nucleotide substitutions. Mol. Biol. Evol., 3: 418-426

Ortiz-Rivas, B., S. Jaubert-Possamai, S. Tanguy, J.P. Gauthier, D. Tagu and R. Claude, 2012. Evolutionary study of duplications of the miRNA machinery in aphids associated with striking rate acceleration and changes in expression profiles. BMC Evol. Biol., 12: 216

Rajagopal, R., S. Sivakumar, N. Agrawal, P. Malhotra and R.K. Bhatnagar, 2002. Silencing of midgut aminopeptidase $\mathrm{N}$ of Spodoptera litura by double-stranded RNA establishes its role as Bacillus thuringiensis toxin receptor. J. Biol. Chem., 277: 46849-46851

Richter, H., L. Randau and A. Plagens, 2013. Exploiting CRISPR/Cas: interference mechanisms and applications. Int. J. Mol. Sci., 14: $14518-14531$

Scott, J.G., K. Michel, L.C. Bartholomay, B.D. Siegfried, W.B. Hunter, G. Smagghe, K.Y. Zhu and A.E. Douglas, 2013. Towards the element of successful insect RNAi. J. Insect Physiol., 59: 1212-1221

Swevers, L., J. Liu, H. Huvenne and G. Smagghe, 2011. Search for limiting factors in the RNAi pathway in silkmoth tissues and the Bm5 cell line: the RNA-binding proteins R2D2 and Translin. Plos One, 6 e20250

Swevers, L., J.V. Broeck and G. Smagghe, 2013. The possible impact of persistent virus infection on the function of the RNAi machinery in insects: a hypothesis. Front. Physiol., 4: 319

Tamura, K., J. Dudley, M. Nei and S. Kumar, 2007. MEGA4: molecular evolutionary genetics analysis (MEGA) software version 4.0. Mol. Biol. Evol., 24: 1596-1599 
Tian, H.G., H. Peng, Q. Yao, H.X. Chen, Q. Xie, B. Tang and W.Q. Zhang, 2009. Developmental control of a lepidopteran pest Spodoptera exigua by ingestion of bacteria expressing dsRNA of a non-midgut gene. PLoS One, 4: e6225

Tomoyasu, Y., S.C. Miller, S. Tomita, M. Schoppmeier, D. Grossmann and G. Bucher, 2008. Exploring systemic RNA interference in insects: a genome-wide survey for RNAi genes in Tribolium. Genome Biol., 9: R10

Tu, Y.G. and J.H. Zeng, 2010. A method for artificial rearing of common cutworm, Spodoptera litura. Acta Agric. Jiangxi, 22: 87-88

Tuan, S.J., C.C. Lee and H. Chi, 2014. Population and damage projection of Spodoptera litura (F.) on peanuts (Arachishypogaea L.) under different conditions using the age-stage, two-sex life table. Pest Manage. Sci., 70: 805-813

Winston, W.M., C. Molodowitch and C.P. Hunter, 2002. Systemic RNAi in C. elegans requires the putative transmembrane protein SID-1. Science, 295: 2456-2459
Winston, W.M., M. Sutherlin, A.J. Wright, E.H. Feinberg and C.P. Hunter, 2007. Caenorhabditis elegans SID-2 is required for environmental RNA interference. P. Natl. Acad. Sci. USA, 104: 10565-10570

Wu, J.Y., W.Z. Huang and Z.Y. He, 2013. Dendrimers as Carriers for siRNA Delivery and Gene Silencing: A Review. Sci. World J., Article ID 630654,doi: 10.1155/2013/630654

Zhao, H.M., X. Yi, Z. Hu, S.H. Chen, X.L. Dong and L. Gong, 2013. RNAimediated knockdown of catalase causes cell cycle arrest in SL-1 cells and results in low survival rate of Spodoptera litura (Fabricius). PLoS One, 8: e59527

Zhou, J.L., G.R. Zhang and Q. Zhou, 2012. Molecular characterization of cytochrome P450 CYP6B47 cDNAs and 5'-flanking sequence from Spodoptera litura (Lepidoptera: Noctuidae): Its response to lead stress. J. Insect Physiol., 58: 726-736

(Received 07 May 2014; Accepted 09 January 2015) 